\title{
Finite Element Analysis of Disc Insulator Type and Corona Ring Effect on Electric Field Distribution over 230-kV Insulator Strings
}

\author{
${ }^{1}$ E. Akbari, ${ }^{2}$ M. Mirzaie, ${ }^{3}$ A. Rahimnejad and ${ }^{4}$ M.B. Asadpoor \\ Department of ECE, Babol University of Technology, Babol, Iran \\ E-mail: ${ }^{1}$ e.akbari@stu.nit.ac.ir, ${ }^{2}$ mirzaie@ nit.ac.ir, ${ }^{3}$ a.rahimnejad@stu.nit.ac.ir, \\ ${ }^{4}$ mb.asadpoor@stu.nit.ac.ir
}

\begin{abstract}
Insulator strings are widely used in power systems for the dual task of mechanically supporting and electrically isolating the live phase conductors from the support tower. However, the electric field and voltage distribution of insulator string is uneven which may easily lead to corona, insulators' surface deterioration and even flashover. So the calculation of the electric field and voltage distribution along them is a very important factor in the operation time. Besides, despite the variety of insulator material and profiles, no remarkable endeavor regarding their impacts upon electric field distribution has been made so far. In this paper, three-dimensional Finite Element Method (3-D FEM) software-Maxwell is employed to simulate several $230-\mathrm{kV}$ insulator strings with various types of porcelain and glass disc insulators and the electric field along them were compared, to investigate the effect of insulator types on electric field distribution.
\end{abstract}

Keywords: Electric Field Distribution, Finite Element Method, Porcelain and Glass Insulator Strings

\section{Introduction}

Insulator strings are widely used in power systems for the dual task of mechanically supporting and electrically isolating the live phase conductors from 
the support tower. This is due to their high mechanical strength, easy installation and operation, and low cost.

Due to the coupling capacitance between disc insulators and conductors around them, the potential distribution of insulator string is uneven greatly. The voltage and electric field on the insulators near conductors is three to five times greater than others (without corona ring), which may easily lead to corona, insulators' surface deterioration and even flashover. And these problems will seriously affect the operation safety of transmission lines [1]. So the calculation of the electric field and voltage distribution in and around high voltage insulators is a very important factor in the operation condition. The excessive electric stress on the disc insulators near to energized conductor leads to insulator ageing and surface discharges. Furthermore, the knowledge of the electric field is useful for the detection of defects in insulators [2].

Many publications can be found in the calculation of the E-field and potential distribution for high voltage insulators.

Methods based on field theoretical approach were reviewed in [3], which indicated its advantages in the analysis of electrical insulation problems. However, the electric field evaluation of a practical insulator is so complex that an analytical method is very difficult, if not possible, to compute the exact solutions. Numerical methods are thus often adopted in engineering applications to derive acceptable solutions.

Wei et al. [4] applied charge simulation method (CSM) to calculate potential and electric field distribution along insulator strings, but they simplified the problem which could not include the influence of conductors or towers.

A finite difference method (FDM) to calculate the electric field in and around polluted insulators with asymmetric boundary conditions has been proposed by Morales et al. [5].

Zhao et al. [6] applied boundary element method (BEM) in calculating the potential and electric field distribution along insulators. Their method considered the effects of conductors, grading devices and transmission towers. But computational requirements were high.

Finite element method (FEM) was applied in calculating potential and/or electric field distribution along insulators.

A two-dimensional finite element model for potential and electrical field calculation along an ice-covered ceramic string insulator and composite insulator is presented in [7] and insulators compared with each other. Their calculation results show that the presence of the ice layer and the icicles has significantly influenced on the electric field distribution along the ice-covered composite insulator; and also the maximum electric field along the water films and dry band increases with decreasing the dry-band length. 
In [8] a two-dimensional (2-D) FEM was used for simulation of electric field distribution on ceramic insulators. The voltage applied on insulator has mean effect on electric field; as the voltage value increase the electric field increase. The highest electric field for the insulator exits near the HV end of the insulator.

The electric field distribution of a high voltage suspension insulator has been measured by Kontargyri et al. [9]. In addition, the electric field and potential distribution around and inside the insulator using 3-D FEM model simulated in software OPERA. A very good agreement has been ascertained, by comparing the experimental results with the results from simulation. Reddy et al. [10] used FEMbased software to study the potential and electric field distribution of different types of porcelain disc insulators; but their method was 2-D and could not consider the supporting structures, conductors and other accessories.

Rahimnejad et al. [11] used 3-D FEM for the selection of best corona ring based on their effect on the electric field and voltage distribution of insulator strings.

In this paper several insulator strings with different disc insulators were simulated using a three-dimensional (3-D) electric field program based on finite element method. The electric field and potential distributions along insulators were calculated and compared for different insulator types. The effects of tower and conductor existence were considered. Also the effect of corona ring, for reduction of maximum electric field and improving voltage distribution, was investigated.

\section{The Modeling and Simulation Method}

The finite element method, a numerical calculation method developed rapidly with the development of computer, is applied in electromagnetic field analysis as an important instrument.

\subsection{Main Steps of FEM Implementation}

The main idea of finite element method is "divide and combine", to divide is for the element analyze and to combine is for the sake of synthetically analysis of the integral structure. According to the finite element method, the process of electricfield distribution modeling and calculation are as following:

- Discretization is applied to the calculation model and then nodes and elements are generated. All elements are connected together by the nodes to form the original model.

- Based on the different characteristics of various materials attached to the model, corresponding parameters of electrical characteristics will be defined to each element. 
- Maxwell equations and boundary conditions are used to connect all elements together according to their original structure by proper simplifying and then finite element equations are formed.

- According to the specific feature, proper calculation method is chosen to solve the field physical quantity of unknown nodes. Different solution matrix, which is formed in response to different method, can be solved by iterative method.

In this paper, the surfaces of insulators are clean and dry. Thus, we can solve the case studies by the electrostatic solution.

\subsection{Equations for Electric Field Calculation}

A Simple way for calculation of E-field distribution is calculating of electric potential distribution. Then, E-field distribution is calculated by minus gradient of electric potential distribution. Due to electrostatic field distribution, E-field distribution can be written as follows [12]:

$$
E=-\nabla V
$$

From Maxwell's equation:

$\nabla E=\frac{\rho}{\varepsilon}$

where $\rho$ is resistivity $\Omega / \mathrm{m}$ and $\varepsilon$ is dielectric constant of dielectric material $\left(\varepsilon=\varepsilon_{0} \varepsilon_{\mathrm{r}}\right.$ ). $\varepsilon_{0}$ is air or space dielectric constant $(8.854 \times 10-12 \mathrm{~F} / \mathrm{m}), \varepsilon_{\mathrm{r}}$ is relative dielectric constant of dielectric material. Placing (1) in (2) would give Poisson's equation.

$$
\nabla \varepsilon \nabla V=-\rho
$$

Without space charge $(\rho=0)$, Poisson's equation becomes Laplace's equation.

$$
\nabla \varepsilon \nabla V=0
$$

And finally expansion of (4) for homogeneous media in Cartesian coordinates is:

$$
\frac{\partial^{2} V}{\partial x^{2}}+\frac{\partial^{2} V}{\partial y^{2}}+\frac{\partial^{2} V}{\partial z^{2}}=0
$$

Further details can be achieved in [12]. 


\section{Simulation Parameters}

In this work, porcelain and insulator strings, tower, conductor and corona ring are simulated by Maxwell-Software based on three-dimensional FEM. Insulators and other apparatus characteristics are as follow:

\subsection{Parameters of Insulators, Grading Ring, Tower and Conductors}

The investigations were carried out on three types of porcelain disc insulators and three types of glass disc insulators, which were denominated by type-A to type-F, respectively. Technical parameters of the insulators are introduced in Fig. 1 and given in Table 1, in which $\mathrm{H}$ is the configuration height, $\mathrm{D}$ is the diameter, $\mathrm{L}$ is the leakage distance and Fmin is minimum mechanical failing load. Also profiles of different insulator types are shown in Fig. 2 between them type-D and type-E are fog-type insulators, with greater leakage distance, and others are standard ones.

The length of the conductor, which was used for the simulation of the model, was chosen to be equal to the insulator string length. Tower height was $5000 \mathrm{~mm}$ and cross-arm length was $3175 \mathrm{~mm}$, and finally one of the common types of corona rings in $230-\mathrm{kV}$ power transmission lines was selected for the investigations, whose profile and dimensions are shown in Fig. 3.

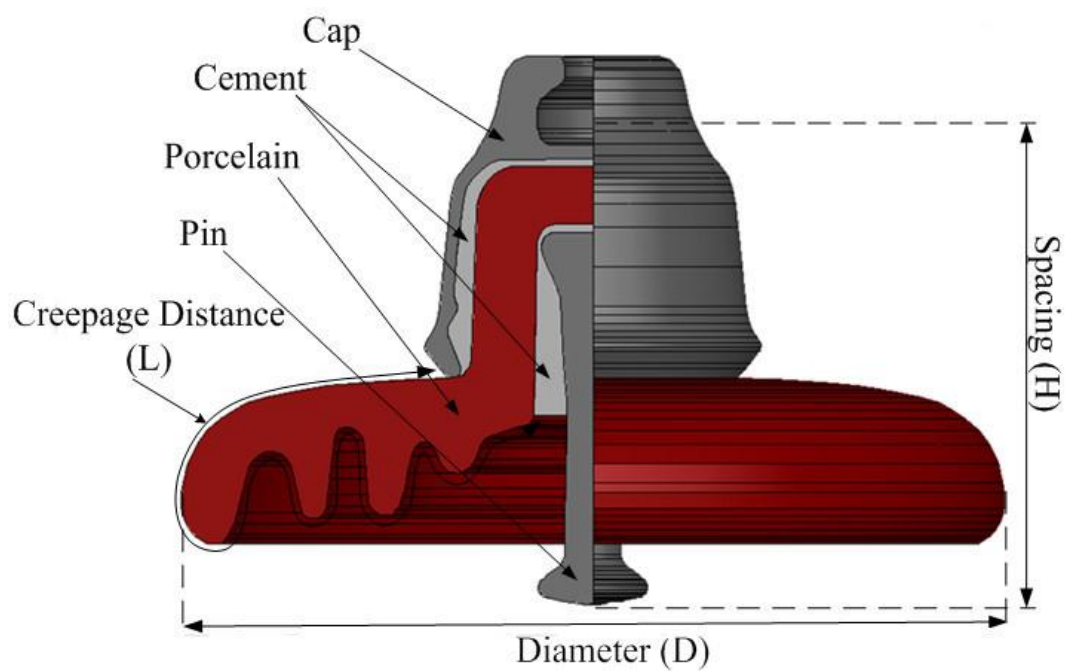

Fig. 1: Dimension parameters and material types of one of the simulated insulators 


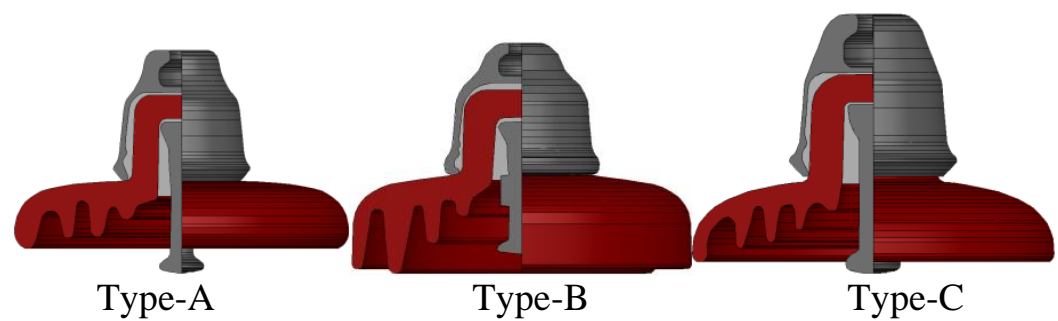

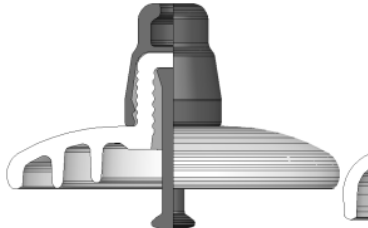

Type-D

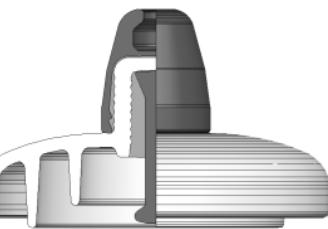

Type-E

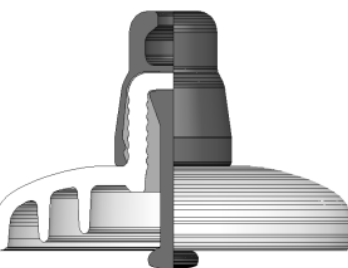

Type-F

Fig. 2: Profiles of the simulated insulators

Table 1: Technical parameters of the simulated insulator

\begin{tabular}{|c|c|c|c|c|c|c|c|}
\hline No. & Type & Material & $\mathrm{H}(\mathrm{mm})$ & $\mathrm{D}(\mathrm{mm})$ & $\mathrm{L}(\mathrm{mm})$ & $\mathrm{F}_{\min }(\mathrm{kN})$ & Manufacturer \\
\hline A & 0207 & Porcelain & 146 & 255 & 295 & 120 & Iran Insulator Co. \\
\hline $\mathrm{B}$ & 0215 & Porcelain & 146 & 255 & 440 & 120 & Iran Insulator Co. \\
\hline $\mathrm{C}$ & 0206 & Porcelain & 170 & 280 & 370 & 160 & Iran Insulator Co. \\
\hline $\mathrm{D}$ & U120B & Glass & 146 & 255 & 320 & 120 & Pars Maghareh Co. \\
\hline $\mathrm{E}$ & U120BP & Glass & 146 & 280 & 442 & 120 & Pars Maghareh Co. \\
\hline $\mathrm{F}$ & U160BL & Glass & 170 & 280 & 370 & 160 & Pars Maghareh Co. \\
\hline
\end{tabular}




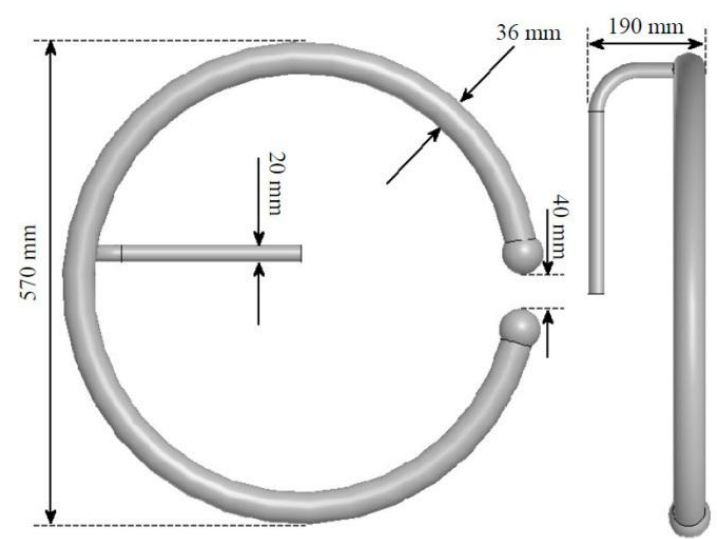

(a)

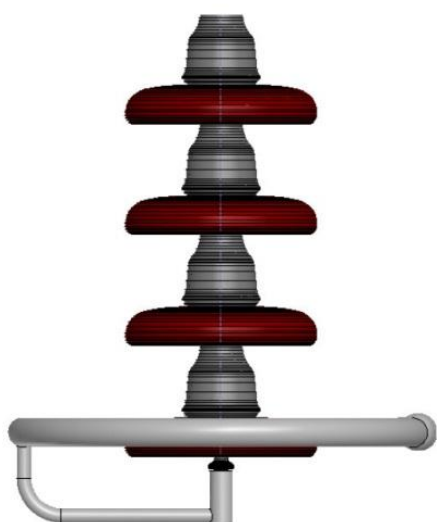

(b)

Fig. 3: Schema of simulated corona ring (a) profile and dimensions (b) installation configuration

\subsection{Simulated Model}

The 3-D model simulated in FEM, which consists of the insulator string, corona ring, the transmission line and also simplified tower, is shown in Fig. 4.

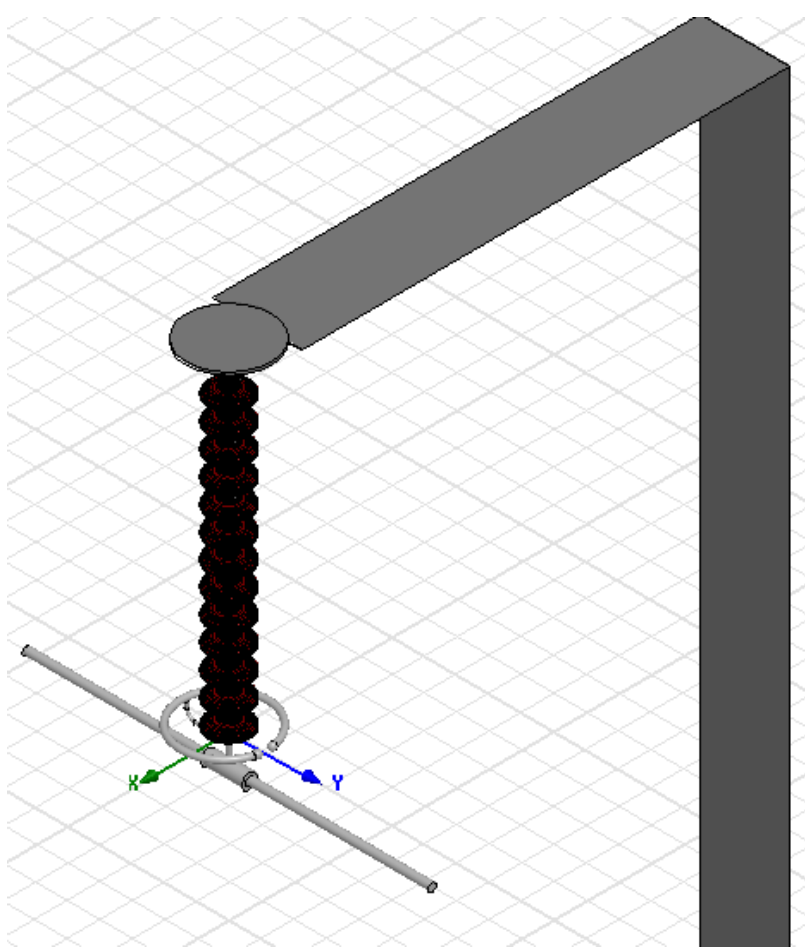

Fig. 4: The model of tower, conductor, and insulator string used for simulation 


\section{Simulation Results}

To investigate the effect of insulator type and profile on electric field, simulation of insulator strings in different conditions has been carried out by software based on FEM and results were presented.

\subsection{Presentation of the results}

Because of the large number of simulations carried out, only some results of typeA and type-D insulator string were extracted from FEM software and is presented here as a sample. Results of other insulator types are discussed through appropriate figures. The electric field distribution on $\mathrm{YZ}$ plane for type-A and type-D insulator strings without and with corona ring are presented in Fig. 5 and 6 , respectively. It is obvious from these figures that the triple junctions (pincement, cement-porcelain) are critical areas where electric field stress is maximum and damage and consequent breakdown may occur, especially for the bottom insulator disc.

In order to study the distribution of electric field in and about insulator string three lines are considered and electric field distribution along them is calculated and presented here.

- Line 1: the line that passes the center of the insulators, the string axis

- Line 2: the line which is parallel to line 1 and touches the insulator surface (located in $\mathrm{x}=0$ and $\mathrm{y}=\mathrm{D} / 2$ )

- Line3: the line which is parallel to line 1 and is $3 * \mathrm{D} / 2 \mathrm{~mm}$ away from it (located in $\mathrm{x}=0$ and $\mathrm{y}=3 * \mathrm{D} / 2$ ).

The electric field plot along lines 1-3 for type-A and type-D insulator string without and with corona ring are shown in Fig. 7 and 8, respectively. In these figures, the electric field along line 1, within metal parts (cap and pin) is zero and sharp local maximums represent the areas where the line passes through cement and porcelain. Along line 2, local maximums of electric field occur in points which the line touches insulator surface. Finally, electric field along line 3, which is completely located in the air, constantly reduces from high voltage terminal side to ground terminal. 


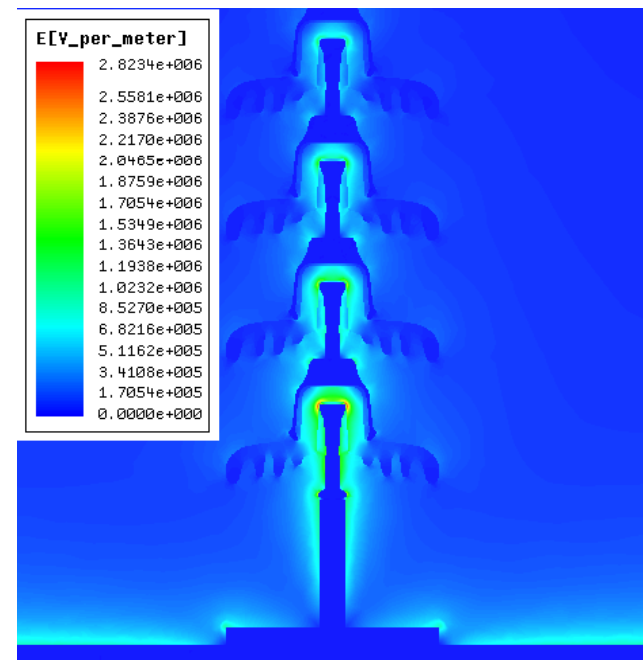

(a)

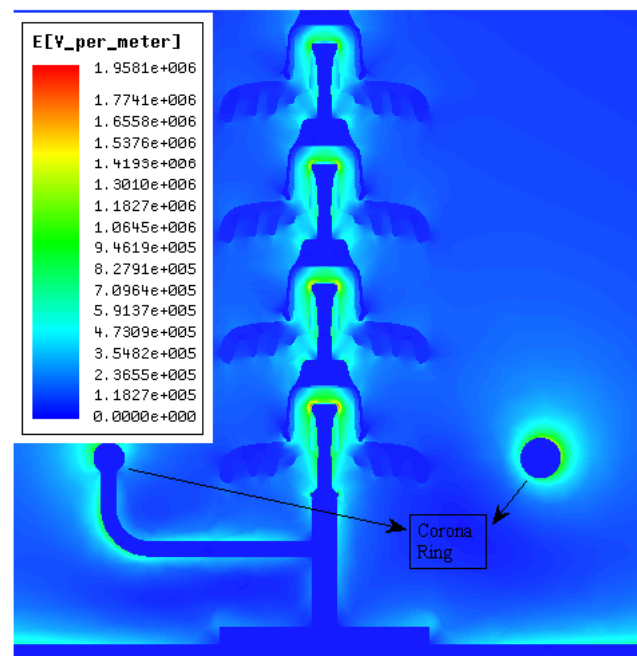

(b)

Fig. 5: Electric field distribution on YZ plane for type-A insulator string (a) without corona ring (b) with corona ring

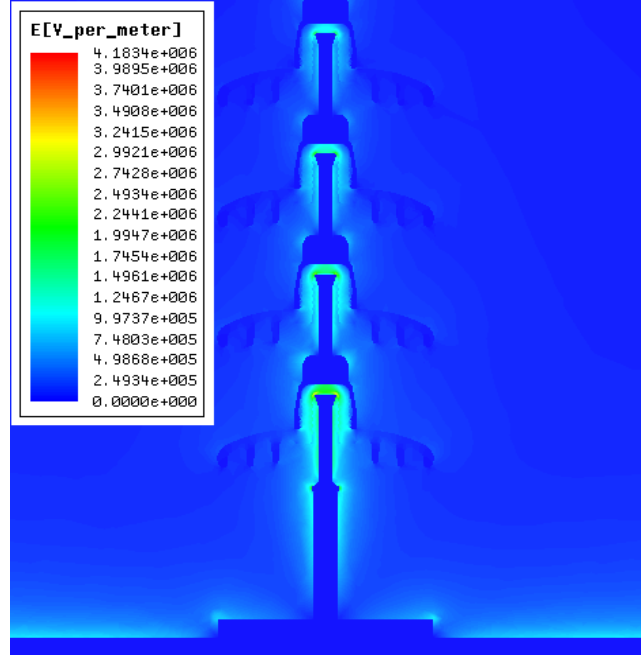

(a)

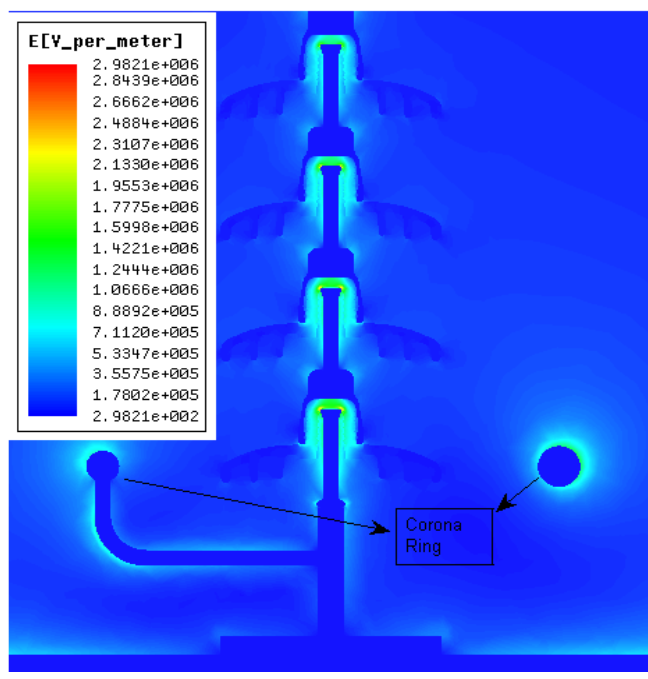

(b)

Fig. 6: Electric field distribution on YZ plane for type-D insulator string (a) without corona ring (b) with corona ring 


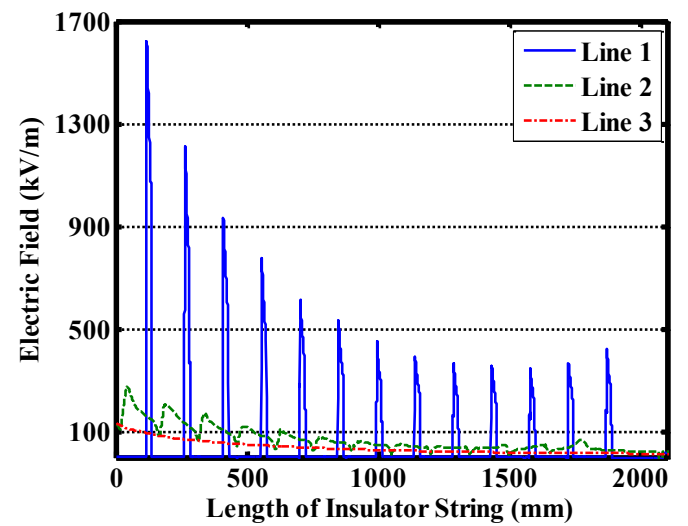

(a)

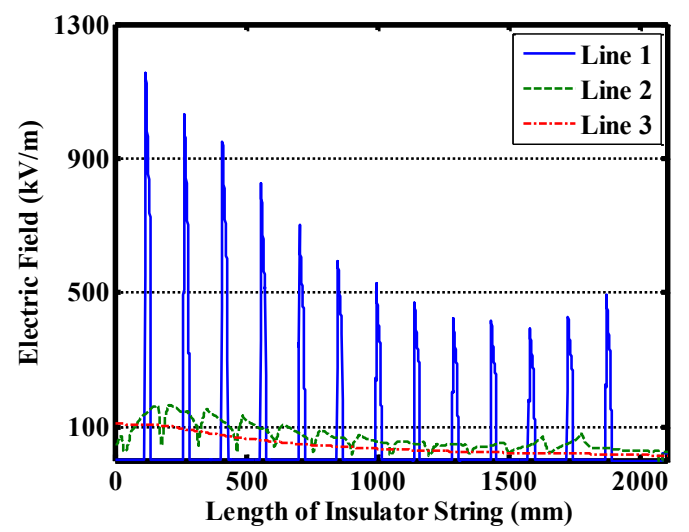

(b)

Fig. 7. Electric field plot on lines 1-3 for type-A insulator string (a) without corona ring (b) with corona ring

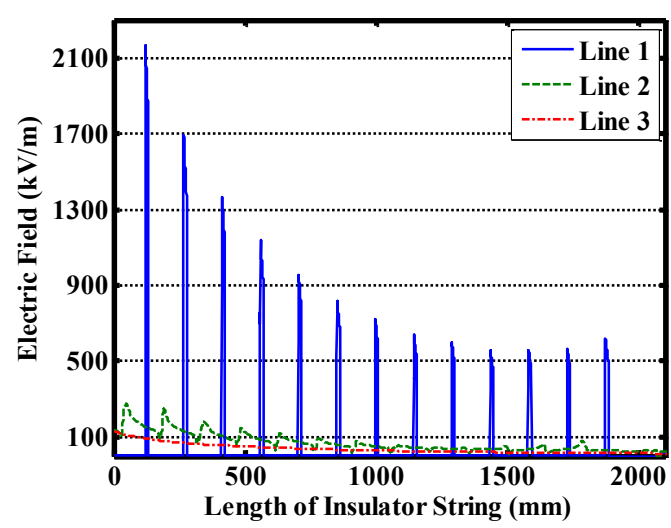

(a)

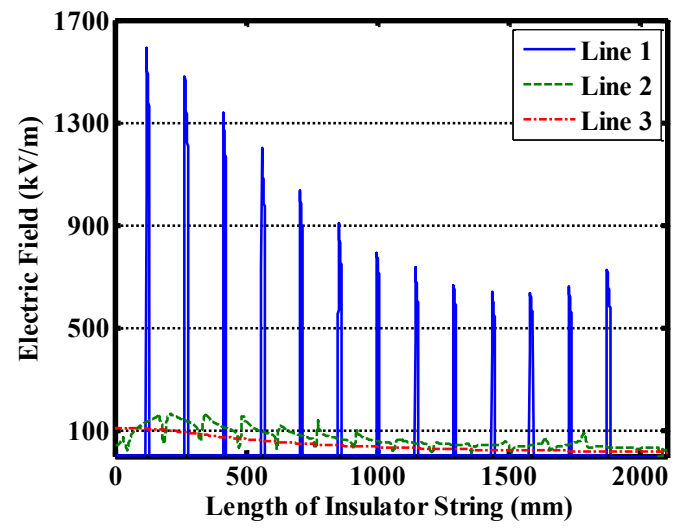

(b)

Fig. 8. Electric field plot on lines 1-3 for type-D insulator string (a) without corona ring (b) with corona ring

\subsection{Effects of Types of Insulator Units}

Local maximums of electric field along line 1 for different insulator strings are given in Fig. 9(a). Besides, absolute maxima of electric field along each of three lines for different types of insulator strings are presented in Fig 10(a). It is obvious from Fig 9(a) that, the bottom insulator which is connected to high voltage conductor bears the maximum electric field which makes it first vulnerable for damage and subsequent breakdown. Also it can be found from these figures that the type of insulator is a determining factor for electric field distribution. Furthermore, it is comprehended that, values of electric field is much higher along glass insulator strings, which is due to higher permittivity of glass. Finally it is deduced from Fig 10(a) that, as the distance from the insulator string increases, the dependency of the electric field to insulator type decreases, i.e. the 
electric field value near insulator string is related to insulator material and profiles, but at farther distances it is not.

\subsection{Effects of Corona Ring}

To investigate the effects of corona ring existence on electric field distribution, the introduced corona ring was added in all of the simulations of the previous section and new results were given in Fig. 9(b) and 10(b), respectively. Comparing these figures with previous ones, it is comprehended that the maximum value of electric field along an insulator string significantly decreases using this apparatus. Furthermore, comparing field values for strings without and with corona ring, and considering dimensions and materials of insulators from Table 1, it can be deduced that the degree of reduction of maximum electric field using corona ring depends on insulator material and profile, as well as the corona ring configuration parameters. Finally, as deduced in previous section, the more distance from insulator string, the less relevant is electric field to insulator type.

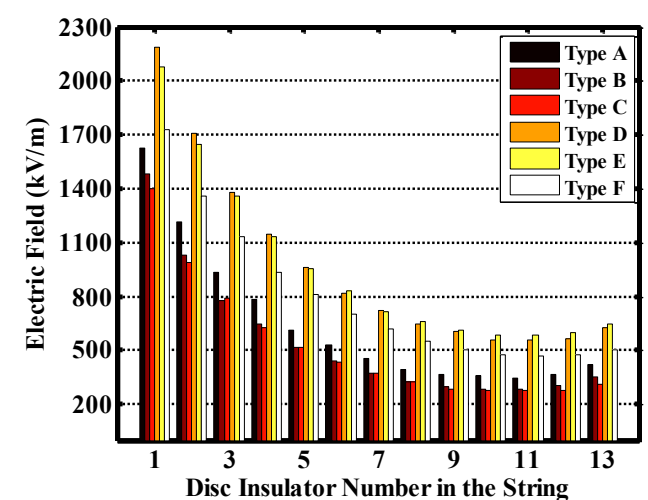

(a)

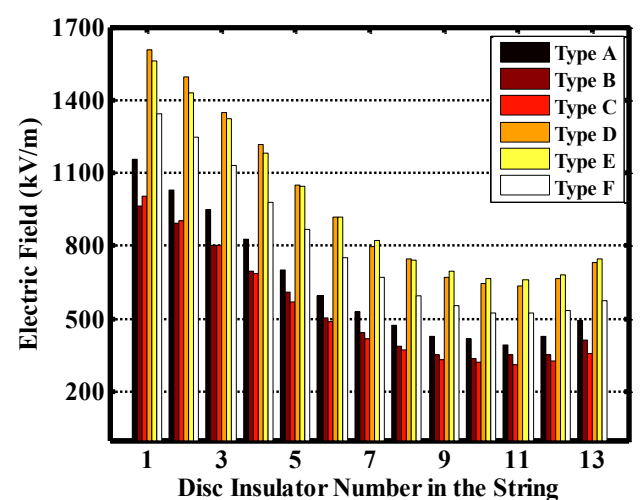

(b)

Fig. 9. Local maximums of electric field along line 1 for different insulator strings (a) without corona ring (b) with corona ring

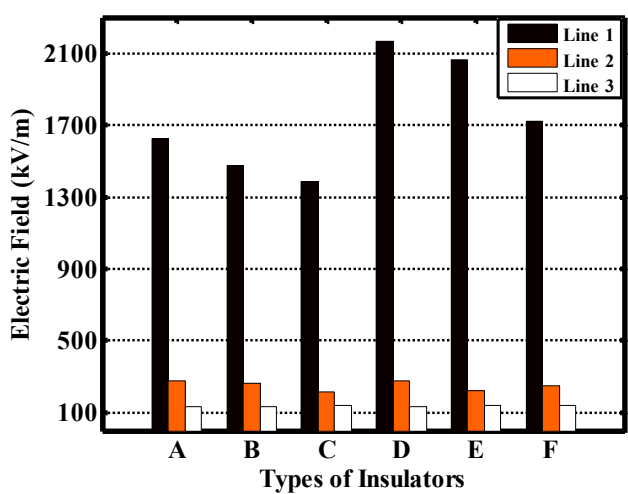

(a)

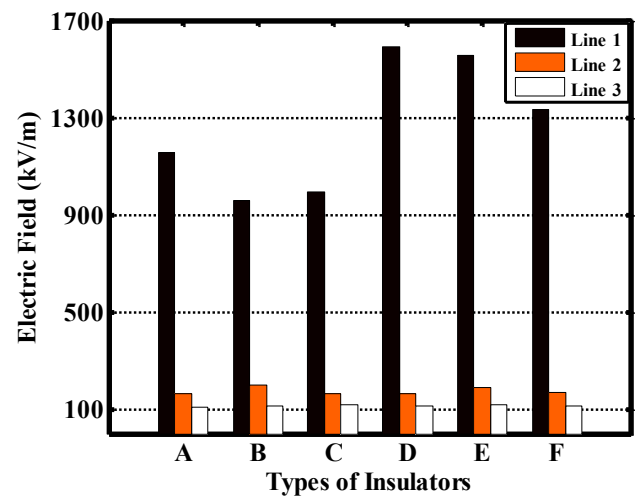

(b)

Fig. 10. Maximum values of electric field along lines 1-3 for all types of insulator strings (a) without corona ring (b) with corona ring 


\section{Conclusions and Discussions}

An attempt was done to understand the effect of disc insulator type and corona ring on the electric field distribution of $230-\mathrm{kV}$ insulator strings. 3-D simulations were carried out using software based on FEM and the effects of nonsymmetrical components such as tower and conductor is taken into account.

Calculations of electric field are useful in identification of vulnerable areas of insulator units where damage and consequent breakdown may occur. From the results presented it is found that one of the most critical areas of electric field stress is triple junction (pin-cement, cement-porcelain/glass) of the bottom disc insulator unit.

According to the results, distribution of electric field over insulator strings without corona ring depends on insulator material and profile, as well as the corona ring configuration parameters. Hence for each particular type of insulator, corona ring parameters should be optimized to give the maximum reduction in the electric field.

Also it was concluded that, as the distance from insulator string increases, the dependency of electric field to insulator material and profile decreases.

All of insulators simulated in this work are common in power system; hence it is believed that the results can be very useful for the manufacturers and utilities.

\section{Acknowledgements}

The authors gratefully acknowledge Iran Insulator Co. and Pars Maghareh Co. for presenting the technical parameters of disc insulators.

\section{References}

[1] B. Wang, ZR. Peng, "A Finite Element Method for the Calculation of the Voltage Distribution along the 500kV Line Insulators", Insulators and Surge Arresters, No.1, (2003), pp.13-15.

[2] V.T. Kontargyri, I.F. Gonos, I.A. Stathopulos, A.M. Michaelides, "Measurement and verification of the voltage distribution on high-voltage insulators", Proceedings of the 12th Biennial IEEE Conference on Electromagnetic Field Computation (CEFC 2006), Maimi, FL.

[3] W. McAllister, "Electric fields and electrical insulation", IEEE Transactions on Dielectrics and Electrical Insulation, Vol. 9, No. 5, (2002), pp. 672-696.

[4] H. Wei, Y. Fan, W. Jingang, Y. Hao, C. Minyou, and Y. Degui, "Inverse application of charge simulation method in detecting faulty ceramic 
insulators and processing influence from tower", IEEE Transactions on Magnetics, Vol. 42, No. 4, (2006), pp. 723-726.

[5] N. Morales, E. Asenj, and A. Valdenegro, "Field solution in polluted insulators with non-symmetric boundary conditions", IEEE Transactions on Dielectrics and Electrical Insulation, Vol. 8, No. 2, (2001), pp. 168-172.

[6] T. Zhao, and M. G. Comber, "Calculation of electric field and potential distribution along nonceramic insulators considering the effects of conductors and transmission towers", IEEE Transactions on Power Delivery, Vol. 15, No. 1, (2000), pp. 313-318.

[7] W. Sima, Q. Yang, C. Sun and F.Guo, "Potential and Electric-Field Calculation along an Ice-Covered Composite Insulator with Finite-Element Method", IEE Proceedings of Generation, Transmission and Distribution, Vol. 153, no. 3, (2006), pp. 343-349.

[8] Sh. M. Faisal, "Simulation of Electric Field Distribution on Ceramic Insulator Using Finite Element Method", European Journal of Scientific Research, Vol.52, No.1, (2011), pp.52-60.

[9] Vassiliki T. Kontargyri, Ioannis F. Gonos and Ioannis A. Stathopulos, "Measurement and simulation of the electric field of high voltage suspension insulators", European Transactions on Electrical Power, Vol. 19, No 3, (2009), pp. 509-517.

[10] B. S. Reddy, N. A. Sultan, P. M. Monika, B. Pooja, O. Salma and K. V. Ravishankar, "Simulation of potential and electric field for high voltage ceramic disc insulators", International Conference on Industrial and Information Systems (ICIIS), Indian Institute of Science, Bangalore, India, (2010), pp. 526-531.

[11] A. Rahimnejad, M. Mirzaie, "Optimal Corona Ring Selection for $230 \mathrm{kV}$ Ceramic I-string Insulator using 3D Simulation", International Journal of Scientific \& Engineering Research, Vol. 3, Issue 7, (2012), pp. 1-6

[12] Haddad and D.F. Warne, Advances in High Voltage Engineering, IET Power and Energy Series 40, The Institution of Engineering and Technology, (2007). 